\title{
On positive solutions of the homogeneous Hammerstein integral equation
}

\author{
Yu. Kh. Eshkabilov, F. H. Haydarov \\ National University of Uzbekistan, Tashkent, Uzbekistan \\ yusup62@mail.ru, haydarov_imc@mail.ru
}

PACS 02.30.Rz

DOI 10.17586/2220-8054-2015-6-5-618-627

In this paper the existence and uniqueness of positive fixed points operator for a nonlinear integral operator are discussed. We prove the existence of a finite number of positive solutions for the Hammerstein type of integral equation. Obtained results are applied to the study of Gibbs measures for models on a Cayley tree.

Keywords: integral equation of Hammerstein type, fixed point of operator, Gibbs measure, Cayley tree.

Received: 2 April 2015

Revised: 18 July 2015

\section{Introduction}

It is well known that integral equations have wide application in engineering, mechanics, physics, economics, optimization, vehicular traffic, biology, queuing theory and so on (see [1-5]). The theory of integral equations is rapidly developing with the help of tools in functional analysis, topology and fixed point theory. Therefore, many different methods are used to obtain the solution of the nonlinear integral equation. Moreover, some methods can be found in Refs. [6-13], to discuss and obtain a solution for the Hammerstein integral equation. In [11], J.Appell and A.S. Kalitvin used fixed point methods and methods of nonlinear spectral theory to obtain a solution for integral equations of the Hammerstein or Uryson type. The existence of positive solutions of abstract integral equations of Hammerstein type is discussed in [9]. In [7], M.A. Abdou, M.M. El-Borai and M.M. El-Kojok discuss the existence and uniqueness of a solution for the nonlinear integral equation of the Hammerstein type with a discontinuous kernel.

In this present paper, we study the solvability of an homogeneous integral equation of the Hammerstein type. An integral equation of the form:

$$
\int_{0}^{1} K(t, u) \Psi(t, f(u)) d u=f(t),
$$

is called the homogeneous Hammerstein integral equation, where $K(t, u)$ is continuous realvalued function defined on $0 \leq t \leq 0,0 \leq u \leq 1, \Psi:[0,1] \times \mathbb{R} \rightarrow \mathbb{R}$ is a continuous function and $f(t)$ is unknown function from $C[0,1]$.

Let $\Psi(t, z), \frac{\partial}{\partial z} \Psi(t, z)$ be continuous and bounded for $t \in[0,1]$ and for all $z$. Then [14], the Hammerstein integral equation (1.1) has a solution, assuming that $\Psi(t, z)$ is a bounded continuous function for $t \in[0,1]$ and $z \in \mathbb{R}$. In this case, the Hammerstein integral equation (1.1) also has a solution [15]. For the necessary details of this theorem and for more results on the Hammerstein integral equation, we refer to Petryshyn and Fitzpatrik [16], Browder [17], Brezis and Browder [18]. 
Recently, the case $\Psi(t, z)=\Psi(z)$ was considered [19]. Let $\Psi(z)$ be a monotonous left-continuous function on $[0,+\infty)$ and $\lim _{x \rightarrow 0} \frac{\Psi(z)}{z}=+\infty, \lim _{x \rightarrow+\infty} \frac{\Psi(z)}{z}=0$. Then, the integral equation of Hammerstein type (1.1) has a solution [19].

In this work, we will consider the following integral equation of Hammerstein type (i.e. in (1.1) $\left.\Psi(t, z)=\Psi(z)=z^{\vartheta}\right)$ :

$$
\int_{0}^{1} K(t, u) f^{\vartheta}(u) d u=f(t), \quad \vartheta>1,
$$

on the $C[0,1]$, where $K(t, u)$ is a strictly positive continuous function.

By Theorem 44.8 from [4], the existence of a nontrivial positive solution for the Hammerstein equation (1.2) follows. We study the problem of the existence of a finite number of positive solutions for the integral equation of the Hammerstein type (1.2).

Consider the nonlinear operator $R_{\alpha}$ on the cone of positive continuous functions on $[0,1]$ :

$$
\left(R_{\alpha} f\right)(t)=\left(\frac{\int_{0}^{1} K(t, u) f(u) d u}{\int_{0}^{1} K(0, u) f(u) d u}\right)^{\alpha},
$$

where $K(t, u)$ is given in the integral equation of Hammerstein type (1.2) and $\alpha>0$. An operator of the form (1.3) arises in the theory of Gibbs measures (see [20-22]). Positive fixed points of the operator $R_{k}, k \in \mathbb{N}$ and their numbers are very important to study Gibbs measures for models on a Cayley tree.

In [21], for the case $\alpha=1$, the uniqueness of positive fixed points of the nonlinear operator $R_{\alpha}$ (1.3) was proved. In [20], in the case $\alpha=k \in \mathbb{N}, k>1$, for the nonlinear operator $R_{\alpha}$, the existence of positive fixed point and the existence Gibbs measure for some mathematical models on a Cayley tree were proved.

The aim of this work is to study the existence of a finite number of positive solutions for the Hammerstein equation (1.2) on the space of continuous functions on $[0,1]$. The plan of this paper is as follows: in the second section, using properties of Hammerstein equation (1.2), we reduce some statements on the positive fixed point of the operator $R_{\alpha}$; in the third section, we construct the strictly positive continuous kernel $K(t, u)$, such that, for given $n \in \mathbb{N}$, the corresponding Hammerstein equation (1.2) has $n$ number of positive Solutions; in the fourth section, the obtained results for the operator $R_{\alpha}$ are applied to study Gibbs measures for models on a Cayley tree.

\section{Existence and uniqueness of positive fixed points for the operator $\boldsymbol{R}_{\alpha}$}

In this section, we study the existence and the uniqueness of positive fixed points for the nonlinear operator $R_{\alpha}(1.3)$. We set:

$$
C^{+}[0,1]=\{f \in C[0,1]: f(x) \geq 0\}, \quad C_{0}^{+}[0,1]=C^{+}[0,1] \backslash\{\theta \equiv 0\},
$$

where the set $C^{+}[0,1]$ is the cone of positive continuous functions on $[0,1]$.

We define the Hammerstein operator $H_{\vartheta}$ on $C[0,1]$ by the equality:

$$
H_{\vartheta} f(t)=\int_{0}^{1} K(t, u) f^{\vartheta}(u) d u=f(t), \quad \vartheta>1 .
$$

Clearly, by Theorem 44.8 from [4], we obtain: 
Theorem 1. Let $\vartheta>1$. The equation:

$$
H_{\vartheta} f=f
$$

has at least one solution in $C_{0}^{+}[0,1]$.

We set:

$$
\mathcal{M}_{0}=\left\{f \in C^{+}[0,1]: f(0)=1\right\} .
$$

Lemma 1. Let $\alpha>1$. The equation

$$
R_{\alpha} f=f, \quad f \in C_{0}^{+}[0,1]
$$

has a positive solution iff the Hammerstein operator has a positive eigenvalue, i.e. the Hammerstein equation:

$$
H_{\alpha} g=\lambda g, \quad f \in C^{+}[0,1]
$$

has a positive solution in $\mathcal{M}_{0}$ for some $\lambda>0$.

Proof. We define the linear operator $W$ and the linear functional $\omega$ on the $C[0,1]$ by the following equalities:

$$
(W f)(t)=\int_{0}^{1} K(t, u) f(u) d u, \quad \omega(f)=\int_{0}^{1} K(0, u) f(u) d u .
$$

Necessariness. Let $f_{0} \in C_{0}^{+}[0,1]$ be a solution of the equation (2.2). We have:

$$
\left(W f_{0}\right)(t)=\omega\left(f_{0}\right) \sqrt[\alpha]{f_{0}(t)}
$$

From this equality, we get:

$$
\left(H_{\alpha} h\right)(t)=\lambda_{0} h(t)
$$

where $h(t)=\sqrt[\alpha]{f_{0}(t)}$ and $\lambda_{0}=\omega\left(f_{0}\right)>0$.

It is easy to see that $h \in \mathcal{M}_{0}$ and $h(t)$ is an eigenfunction of the Hammerstein's operator $H_{\alpha}$, corresponding the positive eigenvalue $\lambda_{0}$.

Sufficiency. Let $h \in \mathcal{M}_{0}$ be an eigenfunction of the Hammerstein's operator $H_{\alpha}$. Then, there is a number $\lambda_{0}>0$ such that $H_{\alpha} h=\lambda_{0} h$. From $h(0)=1$, we get $\lambda_{0}=\left(H_{\alpha} h\right)(0)=$ $\omega\left(h^{\alpha}\right)$. Then:

$$
h(t)=\frac{\left(H_{\alpha} h\right)(t)}{\omega\left(h^{\alpha}\right)} .
$$

From this equality, we get $R_{\alpha} f_{0}=f_{0}$ with $f_{0}=h^{\alpha} \in C_{0}^{+}[0,1]$. This completes the proof.

Theorem 2. The equation (2.2) has at least one solution in $C_{0}^{+}[0,1]$.

Let $\lambda_{0}$ be a positive eigenvalue of the Hammerstein operator $H_{\alpha}, \alpha>1$. Then, there exists $f_{0} \in \mathcal{M}_{0}$ such that $H_{\alpha} f_{0}=\lambda_{0} f_{0}$. We take $\lambda \in(0,+\infty), \lambda \neq \lambda_{0}$. We define function $h_{0}(t) \in C_{0}^{+}[0,1]$ by

Then:

$$
h_{0}(t)=\sqrt[\alpha-1]{\frac{\lambda}{\lambda_{0}}} f_{0}(t), \quad t \in[0,1]
$$

$$
H_{\alpha} h_{0}=H_{\alpha}\left(\sqrt[\alpha-1]{\frac{\lambda}{\lambda_{0}}} f_{0}\right)=\lambda h_{0}
$$

i.e. the number $\lambda$ is an eigenvalue of Hammerstein operator $H_{\alpha}$ corresponding the eigenfunction $h_{0}(t)$. This can be easily verified: if the number $\lambda_{0}>0$ is an eigenvalue of the operator 
On positive solutions of the homogeneous Hammerstein integral equation

$H_{\alpha}, \alpha>1$, then an arbitrary positive number is an eigenvalue of the operator $H_{\alpha}$. Therefore, we have:

Lemma 2. a) Let $\alpha>1$.The equation $R_{\alpha} f=f$ has a nontrivial positive solution iff the Hammerstein equation $H_{\alpha} g=g$ has a nontrivial positive solution.

Let $\alpha>1$. We denote by $N_{\text {fix.p }}\left(H_{\alpha}\right)$ and $N_{\text {fix.p }}\left(R_{\alpha}\right)$ numbers of nontrivial positive solutions of the equations (2.1) and (2.2), respectively.

Theorem 3. Let $\alpha>1$. The equality $N_{\text {fix.p }}\left(H_{\alpha}\right)=N_{\text {fix.p }}\left(R_{\alpha}\right)$ is held.

We denote:

$$
\begin{aligned}
& m=\min _{t, u \in[0,1]} K(t, u), \quad M_{0}=\max _{u \in[0,1]} K(0, u), \\
& M=\max _{t, u \in[0,1]} K(t, u), \quad m_{0}=\min _{u \in[0,1]} K(0, u) .
\end{aligned}
$$

Theorem 4. Let $\alpha>1$. If the following inequality holds:

$$
\left(\frac{M}{m}\right)^{\alpha}-\left(\frac{m}{M}\right)^{\alpha}<\frac{1}{\alpha}
$$

then the homogenous Hammerstein equation (2.1) and the equation (2.2) have a unique nontrivial positive solution.

An analogous theorem was proved for $\alpha=k \in \mathbb{N}, k \geq 2$ in [20] and proof of Theorem 4 is analogously obtained.

\section{The existence of a finite number of positive solutions for the homogeneous Hammerstein equation}

In this section, for a given $n \in \mathbb{N}$, we'll show the existence of $n$ number of positive solutions of homogeneous integral equation of Hammerstein type (1.2).

For all $p, n \in \mathbb{N}$ we define following matrices:

$$
\begin{gathered}
\mathbf{A}_{n}^{(p)}=\left\{\frac{1}{2(2 p+i+j)-3}\left(\frac{1}{2}\right)^{2(2 p+i+j-2)}\right\}_{i, j=\overline{1, n}}, n, p \in \mathbb{N} . \\
\mathbf{B}\left[a_{1}, \ldots, a_{n} ; b_{1}, \ldots b_{n}\right]=\left(\frac{1}{a_{i}+b_{j}}\right)_{i, j=\overline{1, n}}, a_{i}, b_{j}>0 . \\
\mathbf{C}_{n}^{(p)}=B[4 p, 4(p+1), \ldots, 2(p+n-1) ; 1,5, \ldots, 4 n-3] .
\end{gathered}
$$

Lemma 3. [25] Let $n \geq 2$. Then:

$$
\operatorname{det} \mathbf{B}\left[a_{1}, \ldots, a_{n} ; b_{1}, \ldots, b_{n}\right]=\frac{\prod_{1 \leq i<j \leq n}\left[\left(a_{i}-a_{j}\right)\left(b_{i}-b_{j}\right)\right]}{\prod_{i, j=1}^{n}\left(a_{i}+b_{j}\right)} .
$$

Corollary 1. $\operatorname{det} \mathbf{A}_{n}^{(p)}=\left(\frac{1}{2}\right)^{2 n(2 p+n-1)} \operatorname{det} \mathbf{C}_{n}^{(p)}$

Proof. Let $i, j \in 1,2, \ldots, n$. We multiply by $2^{2(p+j-1)}$ the $j$ th column of the matrix $\mathbf{A}_{n}^{(p)}$, after that, we multiply by $2^{2(i-1)}$ the $i$-th row of the matrix obtained. As a result, we get $\mathbf{C}_{n}^{(p)}$. 
Lemma 4. Let $\mathbf{B}^{-1}\left[a_{1}, a_{2}, \ldots, a_{n} ; b_{1}, b_{2}, \ldots, b_{n}\right]=\left\{\beta_{i j}\right\}_{i, j=\overline{1, n}}$ be an inverse matrix of $\mathbf{B}\left[a_{1}, a_{2}, \ldots, a_{n} ; b_{1}, b_{2}, \ldots, b_{n}\right]$. Then:

$$
\beta_{j i}=\frac{\prod_{s=1}^{n}\left(a_{s}+b_{j}\right) \prod_{s=1, s \neq i}^{n}\left(a_{i}+b_{s}\right)}{\prod_{s=1, s \neq j}^{n}\left(b_{j}-b_{s}\right) \prod_{s=1, s \neq i}^{n}\left(a_{i}-a_{s}\right)} .
$$

Proof. Subtracting the $j$ th column of $\mathbf{B}\left[a_{1}, a_{2}, \ldots, a_{n} ; b_{1}, b_{2}, \ldots, b_{n}\right]$ from every other column, we get the following equality:

$$
\begin{aligned}
& \operatorname{det} \mathbf{B}\left[a_{1}, a_{2}, \ldots, a_{n} ; b_{1}, b_{2}, \ldots, b_{n}\right]= \\
& \frac{\prod_{s=1, s \neq j}^{n}\left(b_{j}-b_{s}\right)}{\prod_{s=1}^{n}\left(a_{s}+b_{j}\right)}\left(\begin{array}{ccccccc}
\frac{1}{a_{1}+b_{1}} & \cdots & \frac{1}{a_{1}+b_{j-1}} & 1 & \frac{1}{a_{1}+b_{j+1}} & \cdots & \frac{1}{a_{1}+b_{n}} \\
\frac{1}{a_{2}+b_{1}} & \cdots & \frac{1}{a_{2}+b_{j-1}} & 1 & \frac{1}{a_{2}+b_{j+1}} & \cdots & \frac{1}{a_{2}+b_{n}} \\
& \cdots & & \cdots & & \ldots & \\
\frac{1}{a_{n}+b_{1}} & \cdots & \frac{1}{a_{n}+b_{j-1}} & 1 & \frac{1}{a_{n}+b_{j+1}} & \cdots & \frac{1}{a_{n}+b_{n}}
\end{array}\right) .
\end{aligned}
$$

Next, we subtract from the $j$-th row the $i$-th row for every $j \in\{1,2 \ldots, i-1, i+1, \ldots n\}$. Then, $\operatorname{det} \mathbf{B}\left[a_{1}, \ldots, a_{n} ; b_{1}, \ldots, b_{n}\right]=\frac{\prod_{s=1, s \neq j}^{n}\left(b_{j}-b_{s}\right) \prod_{s=1, s \neq i}^{n}\left(a_{i}-a_{s}\right)}{\prod_{s=1}^{n}\left(a_{s}+b_{j}\right) \prod_{s=1, s \neq i}^{n}\left(a_{i}+b_{s}\right)} \times \operatorname{det} \mathbf{B}^{(i, j)}\left[a_{1}, \ldots, a_{n} ; b_{1}, \ldots, b_{n}\right]$, where $\mathbf{B}^{(j, i)}\left[a_{1}, \ldots, a_{n} ; b_{1}, \ldots, b_{n}\right]$ is the cofactor of the element $\frac{1}{a_{i}+a_{j}}$ in $\mathbf{B}\left[a_{1}, \ldots, a_{n} ; b_{1}, \ldots, b_{n}\right]$, since:

$$
\beta_{j i}=\frac{\operatorname{det} \mathbf{B}^{(i, j)}\left[a_{1}, \ldots, a_{n} ; b_{1}, \ldots, b_{n}\right]}{\operatorname{det} \mathbf{B}\left[a_{1}, \ldots, a_{n} ; b_{1}, \ldots, b_{n}\right]}
$$

This completes the proof.

We let:

$$
\left(\mathbf{A}_{n}^{(p)}\right)^{-1}=\left\{\alpha_{i j}\right\}_{i, j \in \overline{1, n}} .
$$

Remark 1. For each $\alpha_{j i}$ element of $\left(\mathbf{A}_{n}^{(p)}\right)^{-1}$, the following equality holds:

$$
\alpha_{j i}=4^{2 p+i+j-n+1} \cdot \frac{\prod_{s=1}^{n}(4 p+2 s+2 j-3) \prod_{s=1, s \neq j}^{n}(4 p+2 s+2 j-3)}{\prod_{s=1, s \neq j}^{n}(j-s) \prod_{s=1, s \neq i}^{n}(i-s)} .
$$

Proof. By Corollary 1 and Lemma 4 we get:

$$
\begin{gathered}
\alpha_{j i}=4^{2 p+i+j} \cdot \frac{\operatorname{det} \mathbf{B}^{(i, j)}[4 p, 4 p+2, \ldots 4 p+2(n-1) ; 1,3, \ldots 2 n-1]}{\operatorname{det} \mathbf{B}[4 p, 4 p+2, \ldots 4 p+2(n-1) ; 1,3, \ldots 2 n-1]}= \\
4^{2 p+i+j} \cdot \frac{\prod_{s=1}^{n}(4 p+2 s+2 j-3) \prod_{s=1, s \neq i}^{n}(4 p+2 s+2 i-3)}{\prod_{s=1, s \neq j}^{n}(2 j-2 s) \prod_{s=1, s \neq i}^{n}(2 i-2 s)} .
\end{gathered}
$$


Here, we denote

$$
\begin{gathered}
\varphi_{(s, n, p)}(u)=\alpha_{s 1} u^{2 p-1}+\ldots+\alpha_{s n} u^{2(n+p)-3}, \quad s, n, p \in \mathbb{N}, u \in[0,1] \\
K_{(n, p)}(t, u ; k)=1+\sum_{s=1}^{n}\left(\sqrt[k]{1+t^{2(p+s)-1}}-1\right) \varphi_{(s, n, p)}(u), \quad k \in \mathbb{N}, k \geq 2, t, u \in[0,1] .
\end{gathered}
$$

Remark 2. For the given $k \in \mathbb{N}, k \geq 2$. the following inequality holds:

$$
K_{(n, p)}\left(t-\frac{1}{2}, u-\frac{1}{2} ; k\right) \leq K_{(n, 1)}\left(t-\frac{1}{2}, u-\frac{1}{2} ; k\right), \quad(t, u) \in[0,1]^{2}, n, p \in \mathbb{N} .
$$

We set:

$$
\zeta_{0}(n)=\frac{64}{9} \cdot \frac{4^{n}-1}{4 n+1}\left(\frac{(4 n+1) ! !}{(n-1) !(2 n+1) ! !}\right)^{2} .
$$

Lemma 5. Let $n \in \mathbb{N}$. If $k \geq \zeta_{0}(n)$, then the following inequality holds:

$$
K_{(n, p)}\left(t-\frac{1}{2}, u-\frac{1}{2} ; k\right)>0,(t, u) \in[0,1]^{2}, p \in \mathbb{N} .
$$

Proof. For $p=1$ from Remark 1, we have:

$$
\alpha_{i j}=4^{i+j-n+3} \frac{\prod_{s=1}^{n}(2 i+2 s+1) \prod_{s=1, s \neq j}^{n}(2 j+2 s+1)}{\prod_{s=1, s \neq i}^{n}(i-s) \prod_{s=1, s \neq j}^{n}(j-s)} .
$$

Then:

$$
\left|\frac{\alpha_{i, j+1}}{\alpha_{i, j}}\right|=\frac{4(4 j+1)(2 j+2 n+3)}{(2 j+3)(4 j+5)}, i=\overline{1, n}, j=\overline{1, n-1}
$$

and

$$
\left|\frac{\alpha_{i+1, j}}{\alpha_{i, j}}\right|=\frac{4(n-i)(2 i+2 n+3)}{i(2 i+3)}, i=\overline{1, n}, j=\overline{1, n-1} .
$$

From the above, one has: $\max _{i, j=\overline{1, n}}\left|\alpha_{i j}\right|=\left|\alpha_{n n}\right|$. By Remark 1, we can take:

$$
\begin{gathered}
K_{(n, p)}\left(t-\frac{1}{2}, u-\frac{1}{2} ; k\right) \geq 1-\frac{2}{3} \max _{i, j=1, n}\left|\alpha_{i j}\right| \sum_{s=1}^{n}\left(\sqrt[k]{1+\left(\frac{1}{2}\right)^{2 s+1}-1}\right) \geq \\
1-\frac{2\left|a_{n n}\right|}{3 k} \sum_{s=1}^{n}\left(\frac{1}{2}\right)^{2 s+1} \geq 1-\left(4^{n}-1\right) \cdot \frac{64(2 n+3)^{2}(2 n+5)^{2} \ldots(4 n-1)^{2}(4 n+1)}{9 k((n-1) !)^{2}} .
\end{gathered}
$$

Since $k \geq \zeta_{0}(n)$, one gets $K_{(n, p)}\left(t-\frac{1}{2}, u-\frac{1}{2} ; k\right)>0$. This completes the proof. 
Proposition 1. Let $n \in \mathbb{N}$. If $k \geq \zeta_{0}(n)$, then the Hammerstein's nonlinear operator $H_{k}$ with kernel $K_{(n, p)}\left(t-\frac{1}{2}, u-\frac{1}{2} ; k\right) \quad(p \in \mathbb{N})$ has at least $n$ number of positive fixed points.

Proof. Let $f_{j}(u)=\sqrt[k]{1+u^{2(p+j)-1}}, j=\overline{1, n}$ and $u_{1}=u-\frac{1}{2}, t_{1}=t-\frac{1}{2}$. Put $g_{j}(t)=$ $f_{j}\left(t-\frac{1}{2}\right)$. We will show functions $g_{j}(t)$ are fixed points of the Hammerstein operator $H_{k}$ with the kernel $K_{(n, p)}\left(t-\frac{1}{2}, u-\frac{1}{2} ; k\right)$ :

$$
\begin{gathered}
\int_{0}^{1} K_{(n, p)}\left(t-\frac{1}{2}, u-\frac{1}{2} ; k\right) g_{j}^{k}(u) d u= \\
\int_{0}^{1} K_{(n, p)}\left(t-\frac{1}{2}, u-\frac{1}{2} ; k\right) f_{j}^{k}\left(u-\frac{1}{2}\right) d u=\int_{-\frac{1}{2}}^{\frac{1}{2}} K_{(n, p)}\left(t_{1}, u_{1} ; k\right) f_{j}^{k}\left(u_{1}\right) d u_{1}= \\
\int_{\frac{1}{2}}^{-\frac{1}{2}}\left[+\sum_{s=1}^{n}\left(\sqrt[k]{1+t_{1}^{2(p+s)-1}}-1\right) \varphi_{(s, n, p)}\left(u_{1}\right)\right]\left(1+u_{1}^{2(p+j)-1}\right) d u_{1}= \\
1+\sum_{s=1}^{n}\left(\sqrt[k]{\left.1+t_{1}^{2(p+s)-1}-1\right) \int_{\frac{1}{2}}^{\frac{1}{2}}\left(\alpha_{s 1} u_{1}^{4(p-1)+2 s+2 j}+\ldots+\alpha_{s n} u_{1}^{4 p+2(s+j+n)-6}\right) d u_{1}=}\right. \\
1+\sum_{s=1}^{n}\left(\sqrt[k]{1+t_{1}^{2(p+s)-1}}-1\right)\left(\alpha_{s j} \beta_{s 1}+\ldots+\alpha_{n j} \beta_{s n}\right)=\sqrt[k]{1+t_{1}^{2(p+j)-1}} .
\end{gathered}
$$

Hence:

$$
\int_{0}^{1} K_{(n, p)}\left(t-\frac{1}{2}, u-\frac{1}{2} ; k\right) g_{j}^{k}(u) d u=g_{j}(t), \quad j \in\{1,2, \ldots, n\} .
$$

Theorem 5. For each $n \in \mathbb{N}$, there exists $\vartheta>1$ and a positive continuous kernel $K(t, u)$ such that, the number of positive solutions for the Hammerstein integral equation (1.2) is equal to at least $n$.

\section{Gibbs measures for models on Cayley tree $\Gamma^{k}$}

In this section we study Gibbs measures for models on Cayley tree. You may be familiar with the definitions and properties of Gibbs measures in books [22-24]. A Cayley tree (Bethe lattice) $\Gamma^{k}$ of order $k \in \mathbb{N}$ is an infinite homogeneous tree, i.e., a graph without cycles, such that exactly $k+1$ edges originate from each vertex. Let $\Gamma^{k}=(V, L)$ where $V$ is the set of vertices and $L$ that of edges (arcs). Two vertices $x$ and $y$ are called nearest neighbors if there exists an edge $l \in L$ connecting them. We will use the notation $l=\langle x, y\rangle$. A collection of nearest neighbor pairs $\left\langle x, x_{1}\right\rangle,\left\langle x_{1}, x_{2}\right\rangle, \ldots\left\langle x_{d-1}, y\right\rangle$ is called a path from $x$ to $y$. The distance $d(x, y)$ on the Cayley tree is the number of edges of the shortest path from $x$ to $y$. 
For a fixed $x^{0} \in V$, called the root, we set:

$$
W_{n}=\left\{x \in V \mid d\left(x, x^{0}\right)=n\right\}, \quad V_{n}=\bigcup_{m=0}^{n} W_{m}
$$

and denote:

$$
S(x)=\left\{y \in W_{n+1}: d(x, y)=1\right\}, x \in W_{n},
$$

the set of direct successors of $x$.

Consider models where the spin takes values in the set $[0,1]$, and is assigned to the vertices of the tree. For $A \subset V$ a configuration $\sigma_{A}$ on $A$ is an arbitrary function $\sigma_{A}: A \rightarrow[0,1]$. We denote $\Omega_{A}=[0,1]^{A}$ the set of all configurations on $A$ and $\Omega=[0,1]^{V}$. The Hamiltonian on $\Gamma^{k}$ of the model is:

$$
H(\sigma)=-J \sum_{\langle x, y\rangle \in L} \xi(\sigma(x), \sigma(y)), \quad \sigma \in \Omega
$$

where $J \in R \backslash\{0\}$ and $\xi:(u, v) \in[0,1]^{2} \rightarrow \xi_{u, v} \in \mathbb{R}$ is a given bounded, measurable function.

Let $\lambda$ be the Lebesgue measure on $[0,1]$. On the set of all configurations on $A$ the a priori measure $\lambda_{A}$ is introduced as the $|A|$ fold product of the measure $\lambda$. Here and subsequently, $|A|$ denotes the cardinality of $A$. We consider a standard sigma-algebra $\mathcal{B}$ of subsets of $\Omega=[0,1]^{V}$ generated by the measurable cylinder subsets.

Let $\sigma_{n}: x \in V_{n} \mapsto \sigma_{n}(x)$ be a configuration in $V_{n}$ and $h: x \in V \mapsto h_{x}=\left(h_{t, x}, t \in\right.$ $[0,1]) \in \mathbb{R}^{[0,1]}$ be mapping of $x \in V \backslash\left\{x^{0}\right\}$. Given $n=1,2, \ldots$, consider the probability distribution $\mu^{(n)}$ on $\Omega_{V_{n}}$ defined by:

$$
\mu^{(n)}\left(\sigma_{n}\right)=Z_{n}^{-1} \exp \left(-\beta H\left(\sigma_{n}\right)+\sum_{x \in W_{n}} h_{\sigma(x), x}\right) .
$$

Here, as before, $\sigma_{n}: x \in V_{n} \mapsto \sigma(x)$ and $Z_{n}$ is the corresponding partition function:

$$
Z_{n}=\int_{\Omega_{V_{n}}} \exp \left(-\beta H\left(\widetilde{\sigma}_{n}\right)+\sum_{x \in W_{n}} h_{\widetilde{\sigma}(x), x}\right) \lambda_{V_{n}}\left(\widetilde{\sigma}_{n}\right),
$$

where $\beta=T^{-1}, T>0$ - temperature. The probability distributions $\mu^{(n)}$ are compatible [21] if for any $n \geq 1$ and $\sigma_{n-1} \in \Omega_{V_{n-1}}$ :

$$
\int_{\Omega_{W_{n}}} \mu^{(n)}\left(\sigma_{n-1} \vee \omega_{n}\right) \lambda_{W_{n}}\left(d\left(\omega_{n}\right)\right)=\mu^{(n-1)}\left(\sigma_{n-1}\right) .
$$

Here, $\sigma_{n-1} \vee \omega_{n} \in \Omega_{V_{n}}$ is the concatenation of $\sigma_{n-1}$ and $\omega_{n}$. In this case, there exists [21] a unique measure $\mu$ on $\Omega_{V}$ such that, for any $n$ and $\sigma_{n} \in \Omega_{V_{n}}, \mu\left(\left\{\left.\sigma\right|_{V_{n}}=\sigma_{n}\right\}\right)=\mu^{(n)}\left(\sigma_{n}\right)$. The measure $\mu$ is called the splitting Gibbs measure, corresponding to Hamiltonian (4.1) and function $x \mapsto h_{x}, x \neq x^{0}$.

The following statement describes conditions on $h_{x}$ guaranteeing compatibility of the corresponding distributions $\mu^{(n)}\left(\sigma_{n}\right)$.

Proposition 2. [21] The probability distributions $\mu^{(n)}\left(\sigma_{n}\right), n=1,2, \ldots$, in (4.2) are compatible iff for any $x \in V \backslash\left\{x^{0}\right\}$ the following equation holds: 


$$
f(t, x)=\prod_{y \in S(x)} \frac{\int_{0}^{1} \exp \left(J \beta \xi_{t, u}\right) f(u, y) d u}{\int_{0}^{1} \exp \left(J \beta \xi_{0, u}\right) f(u, y) d u} .
$$

Here and below, $f(t, x)=\exp \left(h_{t, x}-h_{0, x}\right), t \in[0,1]$ and $d u=\lambda(d u)$ is the Lebesgue measure.

We consider $\xi_{t u}$ as a continuous function and we are going to solve equation (4.5) in the class of translation - invariant functions $f(t, x)$ (i.e. $f(t, x)=f(t)$ for all $x \in \Gamma^{k} \backslash\left\{x_{0}\right\}$ ). We'll show that there exists a finite number of translation - invariant Gibbs measures for model (4.1).

For translation - invariant functions, equation (4.5) can be written as:

$$
\left(R_{k} f\right)(t)=f(t), k \in \mathbb{N},
$$

where $K(t, u)=Q(t, u)=\exp \left(J \beta \xi_{t u}\right), f(t) \in C_{0}^{+}[0,1], t, u \in[0,1]$ (see [20,21]).

Consequently, for each $k \in \mathbb{N}, k \geq 2$, the Hammerstein integral equation corresponding to the equation (4.6) has the following form:

$$
\int_{0}^{1} Q(t, u) f^{k}(u) d u=f(t)
$$

By Theorem 3 and Propositions 1and 2 we'll obtain the following Theorem:

Theorem 6. Let $n \in \mathbb{N}$. If $k \geq \zeta_{0}(n)$, then number of translation-invariant Gibbs measures for the model:

$$
H(\sigma)=-\frac{1}{\beta} \sum_{<x, y>} \ln \left(K_{(n, p)}\left(\sigma(x)-\frac{1}{2}, \sigma(y)-\frac{1}{2} ; k\right)\right), \quad \sigma \in \Omega(p \in \mathbb{N}),
$$

on the Cayley tree $\Gamma^{k}$ is equal to at least $n$.

\section{References}

[1] M.A. Abdou, A.A. Badr. On a method for solving an integral equation in the displacement contact problem. J. Appl. Math Comput., 2002, 127, P. 65-78.

[2] R. Grimmer, J.H. Liu. Singular perturbations in viscoelasticity. Rocky Mountain J. Math., 1994, 24, P. 61-75.

[3] J.B. Keller, W.E. Olmstead. Temperature of nonlinearly radiating semi-infinite solid. Quart. Appl. Math., 1972, 29, P. 559-566.

[4] M.A. Krasnoselskii, P.P. Zabrejko. Geometrical methods of Nonlinear Analysis. Springer-Verlag, 1984.

[5] W.E. Olmstead, R.A. Handelsman. Diffusion in a semi-infinite region with nonlinear surface dissipation. SIAM Rev., 1996, 18, P. 275-291.

[6] M.A. Abdou. On the solution of linear and nonlinear integral equation. J. Appl. Math. Comput., 2003, 146, P. 857-871.

[7] M.A. Abdou, M.M. El-Borai, M.M. El-Kojok. Toeplitz matrix method and nonlinear integral equation of Hammerstein type. Journal of Computational and Applied Mathematics, 2009, 223, P. 765-776.

[8] M.A. Abdou, W.G. El-Sayed, E.I. Deebs. A solution of nonlinear integral equation. J. Appl. Math. Comput., 2005, 160, P. 1-14.

[9] A. Horvat-Marc. Positive solutions for nonlinear integral equations of Hammerstein type. Carpathian J. Math., 2008, 24 (2), P. 54-62.

[10] J.A. Appell, E. de Pascale, P.P. Zabrejko. On the unique solvability of Hammerstein integral equations with non-symmetric kernels. Progress in Nonlinear Differential Equations and Their Applications, 2000, 40, P. 27-34.

[11] J. Appell, A.S. Kalitvin. Existence results for integral equations: spectral methods VS. Fixed point theory, 2006, 7 (2), P. 219-234. 
[12] D. Bugajewski. On BV-Solutions of some nonlinear integral equations. Integral Equations Operator Theory, 2003, 46, P. 387-398.

[13] P.S. Milojevic. Solvability and the number of solutions of Hammerstein equations. Electronic J. Diff. Equat., 2004, 54, P. 1-25.

[14] H.K. Pathak, G.S. Jeong, Y.J. Cho. Recent fixed point theorems and their applications. Proceedings of nonlinear functional analysis and applications, 1997, 2, P. 89-114.

[15] P. Kumlin. A note on fixed point theory. Functional Analysis. Mathematics, Chalmers $\wp$ GU, 2003/2004, TMA 401/MAN 670.

[16] W.V. Petryshyn, P.M. Fitzpatrik. New existence theorems for nonlinear integral equations of Hammerstein type. Trams. Amer. Math. Soc., 1970, 160, P. 39-63.

[17] F.E. Browder. Nonlinear functional analysis and nonlinear integral equations of Hammerstein and Urysohn type. Contributions to Nonlinear Functional Analysis (ed. C.H. Zarantonello, Academic Press), 1971, P. 99154.

[18] H. Brezis, F.E. Browder. Nonlinear integral equations and systems of Hammerstein type. Advances in Math, 1975, 18, P. 115-147.

[19] E.Yu. Elenskaya. The existence fixed point of monoton left-continuous operators in space with right cone. Izvestiya vuzov. Matematika, 2011, 10, P. 40-47 [in Russian].

[20] Yu.Kh. Eshkabilov, F.H. Haydarov, U.A. Rozikov. Uniqueness of Gibbs Measure for Models With Uncountable Set of Spin Values on a Cayley Tree. Math. Phys. Anal. Geom., 2013, 16 (1), P. 1-17.

[21] Yu.Kh. Eshkabilov, U.A. Rozikov. On models with uncountable set of spin values on a Cayley tree: Integral equations. Math. Phys. Anal. Geom., 2010, 13, P. 275-286.

[22] U.A. Rozikov. Gibbs measures on a Cayley trees. World Sci. Pub, Singapore, 2013.

[23] H.O. Georgii. Gibbs measures and phase transitions. Second edition, de Gruyter Studies in Mathematics, 9. Walter de Gruyter, Berlin, 2011.

[24] C. Preston. Gibbs states on countable sets. Cambridge University Press, London, 1974.

[25] I.V. Proskuryakov. Problems in Linear Algebra. MIR Publishers Moscow, 1978 (English translation). 